\title{
Using Globular Clusters to Test Newton's Law of Gravity
}

\author{
Riccardo Scarpa, Gianni Marconi \& Roberto Gilmozzi \\ European Southern Observatory, Santiago, Chile.
}

\begin{abstract}
New measurements of the velocity dispersion of the globular cluster $\omega$ Centauri allow us to trace its gravitational potential down to an acceleration of $8 \times 10^{-9} \mathrm{~cm} \mathrm{~s}^{-2}$. It is found that the dispersion profile remains flat well inside the tidal radius as soon as the acceleration of gravity approaches $a_{0}$, a result that finds its simplest explanation within the context of MOND. A similar behaviour is observed in the globular cluster M15 showing this is not a peculiar feature of $\omega$ Centauri. This result is surprising and suggestive of a failure of Newton's law at low accelerations.
\end{abstract}

\section{Introduction and Discussion}

We present the results of a study of the kinematics of the external regions of $\omega$ Centauri and M15, a work triggered by the increasing evidence that Newton's law of gravity may not apply to accelerations smaller than $a_{0}=1.2 \times 10^{-8}$ $\mathrm{cm} \mathrm{s}^{-2}$ (Begeman et al. 1991), as proposed within the context of the modified Newtonian dynamics (MOND; Milgrom 1983). We focused on globular clusters because they are believed to be dark matter-free and hence should agree precisely with Newton's law at any acceleration. Interestingly, in $\omega$ Centauri the velocity dispersion does not show the typical Keplerian fall-off, remaining constant at large radii (Fig. 1). It is worth pointing out that assuming a mass-to-light ratio $M / L=1$ in solar units, appropriate for globular clusters as suggested by dynamical studies (Mandushev et al. 1991), it turns out that the dispersion flattens for $a=(2.1 \pm 0.5) \times 10^{-8} \mathrm{~cm} \mathrm{~s}^{-2}$, similar to $a_{0}$. A similar set of data for M15 (Drukier 1998) shows that also in this globular cluster the velocity dispersion profile remains flat (within errors) at large radii (Fig. 1). The flattening of the velocity dispersion occurs for $a=(1.7 \pm 0.6) \times 10^{-8} \mathrm{~cm} \mathrm{~s}^{-2}$, again similar to $a_{0}$.

Though it is conceivable the observed flattening of the velocity dispersion profile is due to either tidal heating or to a massive dark matter halo surrounding the clusters, both scenarios require ad hoc assumptions to make the profile flat. What is striking is that these two clusters have very little in common, having different masses, different positions and different orbits in the galactic halo. Their dynamical evolution was also different to such an extent that while M15 is a textbook example of a globular cluster, $\omega$ Centauri has been claimed to be the merger of two clusters (Lee et al. 1999) or the remnant of a dwarf galaxy (Hilker 2000). Thus we find the fact that the two profiles are so similar a significant one. Globular clusters are hundreds of times smaller and thousands of time less massive than galaxies; nonetheless the velocity dispersion profile of at least these 
two clusters precisely mimics, both in shape and absolute acceleration, that of elliptical galaxies (explained by invoking dark matter; Carollo et al. 1995). There is no reason for the flattening in globular clusters and galaxies to occur for the same value of the acceleration. Therefore, our result finds its simplest explanation within the framework of MOND, supporting the suggestion that Newton's law of gravity may fail in the low acceleration limit.

\section{References}

Begeman K.G., Broeils A.H. \& Sanders R. H. 1991, MNRAS, 249, 523

Carollo C.M., de Zeeuw P.T., van der Marel R.P., et al. 1995, ApJ, 441, L25

Drukier, G.A., Slavin, S.D., Cohn, H.N. et al 1998, ApJ, 115, 708

Hilker M. \& Richtler T. 2000, A\&A, 362, 895

Lee Y.W., Joo J.M., Sohn Y.J. et al. 1999, Nature, 402, 55

Mandushev G., Spassova N. \& Staneva A. 1991, A\&A, 252, 94

Meylan G. \& Mayor M. 1986, A\&A, 166, 122

Meylan G., Mayor M., Duquennoy A. \& Dubath P. 1995, A\&A, 303, 761

Milgrom M. 1983, ApJ, 270, 365

van Leeuwen F., Le Poole R. S., Reijns R.A. et al. 2000, A\&A, 360, 472
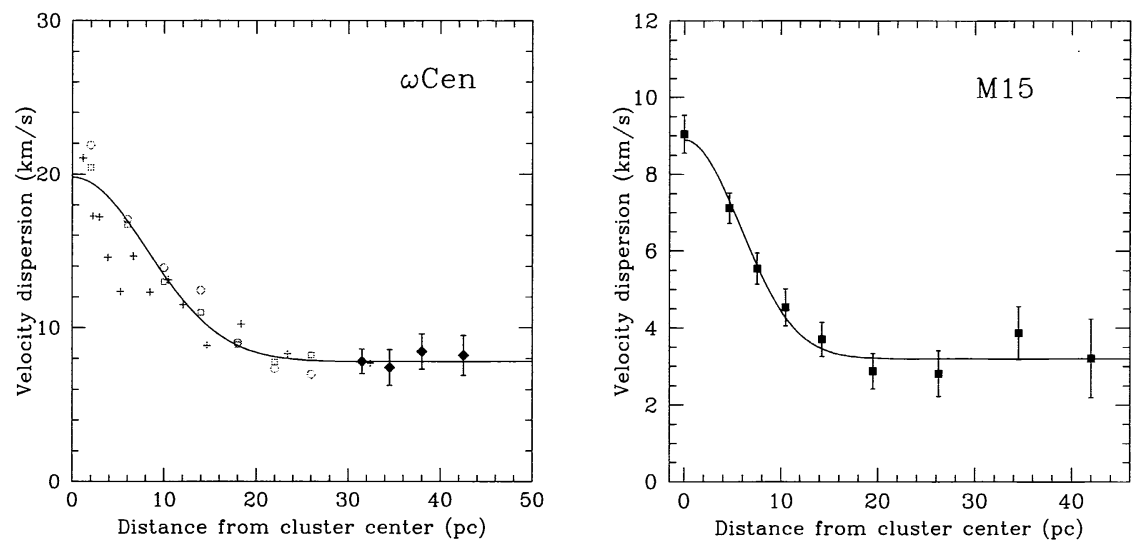

Fig 1. Left: The velocity dispersion profile of $\omega$ Centauri. Proper motion (circles and squares, van Leeuwen et al. 2000) and radial velocities (crosses, Meylan et al. 1995 and Meylan \& Mayor 1986) data show the velocity ellipsoid is isotropic. Our radial velocity measurements (diamonds) extend the profile to $45 \mathrm{pc}$ showing the velocity dispersion remains constant for $\mathrm{R}>27 \pm 3 \mathrm{pc}$. Right: Dispersion profile of the globular cluster M15, from data by Drukier et al. (1998). Also in this case the velocity dispersion remains basically constant for $\mathrm{R}>18 \pm 3 \mathrm{pc}$. In both panels the solid line is not a fit to the data. It is meant to illustrate that the velocity dispersion remains constant at large radii. 